\title{
Existence and Uniqueness of Positive Solutions to Nonlinear Second Order Impulsive Differential Equations with Concave or Convex Nonlinearities
}

\author{
Lingling Zhang ${ }^{1}$ and Chengbo Zhai ${ }^{2}$ \\ ${ }^{1}$ Department of Mathematics, Taiyuan University of Technology, Taiyuan, Shanxi 030024, China \\ ${ }^{2}$ School of Mathematical Sciences, Shanxi University, Taiyuan, Shanxi 030006, China
}

Correspondence should be addressed to Lingling Zhang; zhangllgd@sohu.com

Received 29 January 2013; Accepted 15 May 2013

Academic Editor: Yanbin Sang

Copyright (C) 2013 L. Zhang and C. Zhai. This is an open access article distributed under the Creative Commons Attribution License, which permits unrestricted use, distribution, and reproduction in any medium, provided the original work is properly cited.

Using a new fixed point theorem of generalized concave operators, we present in this paper criteria which guarantee the existence and uniqueness of positive solutions to nonlinear two-point boundary value problems for second-order impulsive differential equations with concave or convex nonlinearities.

\section{Introduction}

In this paper, we study the existence and uniqueness of positive solutions to the following two-point boundary value problems for second-order impulsive differential equations:

$$
\begin{aligned}
& -x^{\prime \prime}(t)=f(t, x(t)), \quad t \in J, \quad t \neq t_{k}, k=1,2, \ldots, m, \\
& \left.\Delta x\right|_{t=t_{k}}=I_{k}\left(x\left(t_{k}\right)\right), \quad k=1,2, \ldots, m, \\
& \left.\Delta x^{\prime}\right|_{t=t_{k}}=\bar{I}_{k}\left(x\left(t_{k}\right)\right), \quad k=1,2, \ldots, m, \\
& x(0)=x^{\prime}(0), \quad x(1)=x^{\prime}(1),
\end{aligned}
$$

where $f \in C[J \times \mathbf{R}, \mathbf{R}], J=[0,1], 0<t_{1}<t_{2}<\cdots<t_{k}<$ $\cdots<t_{m}<1,\left.\Delta x\right|_{t=t_{k}}=x\left(t_{k}^{+}\right)-x\left(t_{k}^{-}\right),\left.\Delta x^{\prime}\right|_{t=t_{k}}=x^{\prime}\left(t_{k}^{+}\right)-x^{\prime}\left(t_{k}^{-}\right)$, $x^{\prime}\left(t_{k}^{+}\right), x^{\prime}\left(t_{k}^{-}\right), x\left(t_{k}^{+}\right), x\left(t_{k}^{-}\right)$denote the right limit (left limit) of $x^{\prime}(t)$ and $x(t)$ at $t=t_{k}$, respectively. $I_{k}, \bar{I}_{k} \in C[\mathbf{R}, \mathbf{R}], k=$ $1,2, \ldots, m$.

Impulsive differential equations have been studied extensively in recent years. Such equations arise in many applications such as spacecraft control, impact mechanics, chemical engineering, and inspection process in operations research. It is now recognized that the theory of impulsive differential equations is a natural framework for a mathematical modelling of many natural phenomena. There have appeared numerous papers on impulsive differential equations during the last ten years. Many of them are on boundary value problems, see [1-18], and it is interesting to note that some of them are about comparatively new applications like ecological competition, respiratory dynamics, and vaccination strategies, see [12, 19-25].

Second-order impulsive differential equations have been studied by many authors with much of the attention given to positive solutions. For a small sample of such work, we refer the reader to works by Feng and Xie [6], Hu et al. [8], Jankowski [10, 11], E. K. Lee and Y.-H. Lee [12], Lin and Jiang [13], Liu et al. [14], Agarwal and O’Regan [26], Wang et al. [27], Zhang [28], and Chu et al. [29]. The results of these papers are based on the Schauder fixed point theorem, Leggett-Williams theorem, fixed point index theorems in cones, Krasnoselski fixed point theorem, the method of upper-lower solutions, fixed point theorems in cones, and so on. But, in most of the existing works, in order to establish the existence of positive solutions, a key condition is the existence of upper-lower solutions. However, as we know, it is difficult to verify the existence of upper-lower solutions for concrete impulsive differential equations. In addition, few papers can be found in the literature on the existence and uniqueness of positive solutions for second-order impulsive differential equations. In this paper, we will study the problem (1) with concave or convex nonlinearities and not suppose 
the existence of upper-lower solutions and compactness condition. Different from the previously mentioned works, in this paper we will use a new fixed point theorem of generalized concave operators to show the existence and uniqueness of positive solutions for the problem (1).

For convenience, we list the following assumptions on the functions $f(t, x), I_{k}(x)$, and $\bar{I}_{k}(x)$ :

$\left(H_{1}\right) f(t, 0) \leq 0, f(t, 1 / 2)<0, t \in[0,1]$, and $f(t, x)$ is decreasing in $x \in[0, \infty)$ for each $t \in[0,1]$,

$\left(H_{2}\right) I_{k}(0) \leq 0, \bar{I}_{k}(0) \geq 0$, and $I_{k}(x)$ is decreasing, and $\bar{I}_{k}(x)$ is increasing in $x \in[0, \infty), k=1,2, \ldots, m$,

$\left(H_{3}\right)$ for any $\lambda \in(0,1), t \in[0,1]$, and $x \geq 0$, there exist $\alpha_{1}(\lambda), \alpha_{2}(\lambda), \alpha_{3}(\lambda) \in(\lambda, 1)$ such that

$$
\begin{gathered}
f(t, \lambda x) \leq \alpha_{1}(\lambda) f(t, x), \\
I_{k}(\lambda x) \leq \alpha_{2}(\lambda) I_{k}(x), \\
\bar{I}_{k}(\lambda x) \geq \alpha_{3}(\lambda) \bar{I}_{k}(x), \\
k=1,2, \ldots, m,
\end{gathered}
$$

$\left(H_{4}\right) \sum_{k=1}^{m}\left[-2 I_{k}(3 / 2)+\left(1+t_{k}\right) \bar{I}_{k}(3 / 2)\right]>0$,

$\left(H_{1}\right)^{\prime} f(t, 3 / 2)<0, t \in[0,1]$, and $f(t, x)$ is increasing in $x \in[0, \infty)$ for each $t \in[0,1]$ and $f(t, x) \leq 0$ for $[0,1] \times[0, \infty)$

$\left(H_{2}\right)^{\prime} I_{k}(x) \leq 0, \bar{I}_{k}(x) \geq 0$ for $[0, \infty)$, and $I_{k}(x)$ is increasing, and $\bar{I}_{k}(x)$ is decreasing in $x \in[0, \infty)$, $k=1,2, \ldots, m$,

$\left(H_{3}\right)^{\prime}$ for any $\lambda \in(0,1), t \in[0,1]$, and $x \geq 0$, there exist $\beta_{1}(\lambda), \beta_{2}(\lambda), \beta_{3}(\lambda) \in(0,1)$ such that

$$
\begin{gathered}
f(t, \lambda x) \geq \lambda^{-\beta_{1}(\lambda)} f(t, x), \\
I_{k}(\lambda x) \geq \lambda^{-\beta_{2}(\lambda)} I_{k}(x), \\
\bar{I}_{k}(\lambda x) \leq \lambda^{-\beta_{3}(\lambda)} \bar{I}_{k}(x), \\
k=1,2, \ldots, m, \\
\left(H_{4}\right)^{\prime} \sum_{k=1}^{m}\left[-2 I_{k}(1 / 2)+\left(1+t_{k}\right) \bar{I}_{k}(1 / 2)\right]>0 .
\end{gathered}
$$

\section{Preliminaries}

In this section, we state some definitions, notations, and known results. For convenience of readers, we suggest that one refers to [30] and references therein for details.

Suppose that $E$ is a real Banach space which is partially ordered by a cone $P \subset E$. That is, $x \leq y$ if and only if $y-x \in P$. By $\theta$ we denote the zero element of $E$. Recall that a nonempty closed convex set $P \subset E$ is called a cone if it satisfies (i) $x \in$ $P, \lambda \geq 0 \Rightarrow \lambda x \in P$, (ii) $x \in P,-x \in P \Rightarrow x=\theta$.

Moreover, $P$ is called normal if there exists a constant $N>$ 0 such that, for all $x, y \in E, \theta \leq x \leq y$ implies $\|x\| \leq N\|y\|$. In this case $N$ is called the normality constant of $P$. We say that an operator $A: E \rightarrow E$ is increasing (decreasing) if $x \leq y$ implies $A x \leq A y(A x \geq A y)$.
For all $x, y \in E$, the notation $x \sim y$ means that there exist $\lambda>0$ and $\mu>0$ such that $\lambda x \leq y \leq \mu x$. Clearly, $\sim$ is an equivalence relation. Given $h>\theta$ (i.e., $h \geq \theta$ and $h \neq \theta$ ), we denote by $P_{h}$ the set $P_{h}=\{x \in E \mid x \sim h\}$. Clearly, $P_{h} \subset P$ is convex and $\lambda P_{h}=P_{h}$ for all $\lambda>0$.

We now present a fixed point theorem of generalized concave operators which will be used in the latter proof. See [30] for further information.

Theorem 1 (from [30, Lemma 2.1, and Theorem 2.1]). Let $h>$ $\theta$, and let $P$ be a normal cone. Assume that $\left(D_{1}\right) A: P \rightarrow P$ is increasing and $A h \in P_{h} ;\left(D_{2}\right)$ for any $x \in P$ and $t \in(0,1)$, there exists $\alpha(t) \in(t, 1)$ with respect to $t$ such that $A(t x) \geq$ $\alpha(t) A x$. Then (i) there are $u_{0}, v_{0} \in P_{h}$ and $r \in(0,1)$ such that $r v_{0} \leq u_{0}<v_{0}, u_{0} \leq A u_{0} \leq A v_{0} \leq v_{0}$; (ii) operator equation $x=A x$ has a unique solution in $P_{h}$.

Remark 2. An operator $A$ is said to be generalized concave if $A$ satisfies condition $\left(D_{2}\right)$.

In what follows, for the sake of convenience, let $\mathrm{PC}[J, \mathbf{R}]=\left\{x \mid x: J \rightarrow \mathbf{R}, x(t)\right.$ be continuous at $t \neq t_{k}$ and left continuous at $t=t_{k}, x\left(t_{k}^{+}\right)$exists, $\left.k=1,2, \ldots, m\right\}$, and let $\mathrm{PC}^{1}[J, \mathbf{R}]=\left\{x \in \mathrm{PC}[J, \mathbf{R}] \mid x^{\prime}(t)\right.$ be continuous at $t \neq t_{k}$ and left continuous at $t=t_{k}, x^{\prime}\left(t_{k}^{+}\right)$exists, $\left.k=1,2, \ldots, m\right\}$. Evidently, $\mathrm{PC}[J, \mathbf{R}]$ is a Banach space with the norm $\|x\|_{\mathrm{PC}}=$ $\sup \{|x(t)|: t \in J\}$, and $\mathrm{PC}^{1}[J, \mathbf{R}]$ is a Banach space with the norm $\|x\|_{\mathrm{PC}^{1}}=\sup \left\{\|x\|_{\mathrm{PC}},\left\|x^{\prime}\right\|_{\mathrm{PC}}\right\}$. Let $J^{\prime}=J \backslash\left\{t_{1}, t_{2}, \ldots, t_{m}\right\}$.

Definition 3. A function $x \in \mathrm{PC}^{1}[J, \mathbf{R}] \cap C^{2}\left[J^{\prime}, \mathbf{R}\right]$ is called a solution of the problem (1) if it satisfies problem (1).

Lemma 4. $x \in P C^{1}[J, \mathbf{R}] \cap C^{2}\left[J^{\prime}, \mathbf{R}\right]$ is a solution of the problem (1) if and only if $x \in P C^{1}[J, \mathbf{R}]$ is the solution of the following integral equation:

$$
\begin{aligned}
x(t)= & -\int_{0}^{1} G(t, s) f(s, x(s)) d s-(1+t) \sum_{k=1}^{m} I_{k}\left(x\left(t_{k}\right)\right) \\
& +\sum_{0<t_{k}<t} I_{k}\left(x\left(t_{k}\right)\right)+\sum_{0<t_{k}<t}\left(t-t_{k}\right) \bar{I}_{k}\left(x\left(t_{k}\right)\right) \\
& +(1+t) \sum_{k=1}^{m} t_{k} \bar{I}_{k}\left(x\left(t_{k}\right)\right),
\end{aligned}
$$

where

$$
G(t, s)= \begin{cases}s(1+t), & 0 \leq t \leq s \leq 1 \\ t(1+s), & 0 \leq s \leq t \leq 1\end{cases}
$$

Proof. First suppose that $x \in \operatorname{PC}^{1}[J, \mathbf{R}] \cap C^{2}\left[J^{\prime}, \mathbf{R}\right]$ is a solution of the problem (1). It is easy to see by integration of (1) that

$$
\begin{aligned}
x^{\prime}(t) & =x^{\prime}(0)-\int_{0}^{t} f(s, x(s)) d s+\sum_{0<t_{k}<t}\left[x^{\prime}\left(t_{k}^{+}\right)-x^{\prime}\left(t_{k}\right)\right] \\
& =x^{\prime}(0)-\int_{0}^{t} f(s, x(s)) d s+\sum_{0<t_{k}<t} \bar{I}_{k}\left(x\left(t_{k}\right)\right) .
\end{aligned}
$$


Integrate again, we can get

$$
\begin{aligned}
x(t)= & x(0)+x^{\prime}(0) t-\int_{0}^{t}(t-s) f(s, x(s)) d s \\
& +\sum_{0<t_{k}<t} \bar{I}_{k}\left(x\left(t_{k}\right)\right)\left(t-t_{k}\right)+\sum_{0<t_{k}<t}\left[x\left(t_{k}^{+}\right)-x\left(t_{k}\right)\right] \\
= & x(0)+x^{\prime}(0) t-\int_{0}^{t}(t-s) f(s, x(s)) d s \\
& +\sum_{0<t_{k}<t} \bar{I}_{k}\left(x\left(t_{k}\right)\right)\left(t-t_{k}\right)+\sum_{0<t_{k}<t} I_{k}\left(x\left(t_{k}\right)\right) .
\end{aligned}
$$

Letting $t=1$ in (6) and (7), we find

$$
\begin{aligned}
x^{\prime}(1)= & x^{\prime}(0)-\int_{0}^{1} f(s, x(s)) d s+\sum_{k=1}^{m} \bar{I}_{k}\left(x\left(t_{k}\right)\right), \\
x(1)= & x(0)+x^{\prime}(0)-\int_{0}^{1}(1-s) f(s, x(s)) d s \\
& +\sum_{k=1}^{m} \bar{I}_{k}\left(x\left(t_{k}\right)\right)\left(1-t_{k}\right)+\sum_{k=1}^{m} I_{k}\left(x\left(t_{k}\right)\right) .
\end{aligned}
$$

From the boundary conditions $x(0)=x^{\prime}(0)$, and $x(1)=$ $x^{\prime}(1)$, we have

$$
\begin{aligned}
x(1)= & x(0)-\int_{0}^{1} f(s, x(s)) d s+\sum_{k=1}^{m} \bar{I}_{k}\left(x\left(t_{k}\right)\right), \\
x(1)= & 2 x(0)-\int_{0}^{1}(1-s) f(s, x(s)) d s \\
& +\sum_{k=1}^{m} \bar{I}_{k}\left(x\left(t_{k}\right)\right)\left(1-t_{k}\right)+\sum_{k=1}^{m} I_{k}\left(x\left(t_{k}\right)\right) .
\end{aligned}
$$

Then we obtain

$$
\begin{aligned}
x(0)= & -\int_{0}^{1} s f(s, x(s)) d s+\sum_{k=1}^{m} \bar{I}_{k}\left(x\left(t_{k}\right)\right) \\
& -\sum_{k=1}^{m} \bar{I}_{k}\left(x\left(t_{k}\right)\right)\left(1-t_{k}\right)-\sum_{k=1}^{m} I_{k}\left(x\left(t_{k}\right)\right) .
\end{aligned}
$$

Substituting (10) into (7), we have

$$
\begin{aligned}
x(t)= & -(1+t) \int_{0}^{1} s f(s, x(s)) d s-\int_{0}^{t}(t-s) f(s, x(s)) d s \\
& -(1+t) \sum_{k=1}^{m} I_{k}\left(x\left(t_{k}\right)\right)+\sum_{0<t_{k}<t} I_{k}\left(x\left(t_{k}\right)\right) \\
& +\sum_{0<t_{k}<t}\left(t-t_{k}\right) \bar{I}_{k}\left(x\left(t_{k}\right)\right)+(1+t) \sum_{k=1}^{m} t_{k} \bar{I}_{k}\left(x\left(t_{k}\right)\right) \\
= & -\int_{0}^{1} G(t, s) f(s, x(s)) d s-(1+t) \sum_{k=1}^{m} I_{k}\left(x\left(t_{k}\right)\right) \\
& +\sum_{0<t_{k}<t} I_{k}\left(x\left(t_{k}\right)\right)+\sum_{0<t_{k}<t}\left(t-t_{k}\right) \bar{I}_{k}\left(x\left(t_{k}\right)\right) \\
& +(1+t) \sum_{k=1}^{m} t_{k} \bar{I}_{k}\left(x\left(t_{k}\right)\right) .
\end{aligned}
$$

Thus, the proof of sufficient is complete.
Conversely, if $x$ is a solution of (4). Then we can easily get $\left.\Delta x\right|_{t=t_{k}}=x\left(t_{k}^{+}\right)-x\left(t_{k}^{-}\right)=I_{k}\left(x\left(t_{k}\right)\right)$. Direct differentiation of (4) implies that, for $t \neq t_{k}$,

$$
\begin{aligned}
x^{\prime}(t)= & -\int_{0}^{t}(1+s) f(s, x(s)) d s-t(1+t) f(t, x(t)) \\
& -\int_{t}^{1} s f(s, x(s)) d s+t(1+t) f(t, x(t)) \\
& -\sum_{k=1}^{m} I_{k}\left(x\left(t_{k}\right)\right)+\sum_{0<t_{k}<t} \bar{I}_{k}\left(x\left(t_{k}\right)\right)+\sum_{k=1}^{m} t_{k} \bar{I}_{k}\left(x\left(t_{k}\right)\right) .
\end{aligned}
$$

Further

$$
\begin{gathered}
x^{\prime \prime}(t)=-f(t, x(t)), \\
\left.\Delta x^{\prime}\right|_{t=t_{k}}=x^{\prime}\left(t_{k}^{+}\right)-x^{\prime}\left(t_{k}^{-}\right)=\bar{I}_{k}\left(x\left(t_{k}\right)\right) .
\end{gathered}
$$

So $x \in C^{2}\left[J^{\prime}, \mathbf{R}\right]$ and it is easy to verify that $x(0)=$ $x^{\prime}(0), x(1)=x^{\prime}(1)$, and the lemma is proved.

Define an operator $A: \mathrm{PC}[J, \mathbf{R}] \rightarrow \mathrm{PC}[J, \mathbf{R}]$ by

$$
\begin{aligned}
A x(t)= & -\int_{0}^{1} G(t, s) f(s, x(s)) d s-(1+t) \sum_{k=1}^{m} I_{k}\left(x\left(t_{k}\right)\right) \\
& +\sum_{0<t_{k}<t} I_{k}\left(x\left(t_{k}\right)\right)+\sum_{0<t_{k}<t}\left(t-t_{k}\right) \bar{I}_{k}\left(x\left(t_{k}\right)\right) \\
& +(1+t) \sum_{k=1}^{m} t_{k} \bar{I}_{k}\left(x\left(t_{k}\right)\right) .
\end{aligned}
$$

Lemma 5. $x \in P C^{1}[J, \mathbf{R}] \cap C^{2}\left[J^{\prime}, \mathbf{R}\right]$ is a solution of problem (1) if and only if $x \in P C^{1}[J, \mathbf{R}]$ is a fixed point of the operator A.

\section{Existence and Uniqueness of Positive Solutions for Problem (1)}

In this section, we apply Theorem 1 to study the problem (1), and we obtain a new result on the existence and uniqueness of positive solutions. The method used in this paper is new to the literature and so is the existence and uniqueness result to the second-order impulsive differential equations. This is also the main motivation for the study of (1) in the present work.

Set $\widetilde{P}=\{u \in \operatorname{PC}[J, \mathbf{R}] \mid u(t) \geq 0, t \in J\}$, the standard cone. It is clear that $\widetilde{P}$ is a normal cone in $\operatorname{PC}[J, \mathbf{R}]$ and the normality constant is 1 . Our main result is summarized in the following theorem.

Theorem 6. Assume that $\left(H_{1}\right)-\left(H_{4}\right)$ hold. Then

(i) there exist $u_{0}, v_{0} \in \widetilde{P}_{h}$ such that

$$
\begin{aligned}
u_{0}(t) \leq & -\int_{0}^{1} G(t, s) f\left(s, u_{0}(s)\right) d s-(1+t) \sum_{k=1}^{m} I_{k}\left(u_{0}\left(t_{k}\right)\right) \\
& +\sum_{0<t_{k}<t} I_{k}\left(u_{0}\left(t_{k}\right)\right)+\sum_{0<t_{k}<t}\left(t-t_{k}\right) \bar{I}_{k}\left(u_{0}\left(t_{k}\right)\right)
\end{aligned}
$$




$$
\begin{aligned}
& +(1+t) \sum_{k=1}^{m} t_{k} \bar{I}_{k}\left(u_{0}\left(t_{k}\right)\right), \quad t \in J, \\
v_{0}(t) \geq & -\int_{0}^{1} G(t, s) f\left(s, v_{0}(s)\right) d s-(1+t) \sum_{k=1}^{m} I_{k}\left(v_{0}\left(t_{k}\right)\right) \\
& +\sum_{0<t_{k}<t} I_{k}\left(v_{0}\left(t_{k}\right)\right)+\sum_{0<t_{k}<t}\left(t-t_{k}\right) \bar{I}_{k}\left(v_{0}\left(t_{k}\right)\right) \\
& +(1+t) \sum_{k=1}^{m} t_{k} \bar{I}_{k}\left(v_{0}\left(t_{k}\right)\right), \quad t \in J,
\end{aligned}
$$

(ii) the nonlinear impulsive problem (1) has a unique positive solution $x^{*}$ in $\widetilde{P}_{h} \cap P C^{1}[J, \mathbf{R}]$, where $h(t)=$ $(1 / 2)\left(t^{2}+t+1\right), t \in[0,1]$.

Remark 7. It is easy to see that $1 / 2 \leq h(t) \leq 3 / 2, t \in[0,1]$.

Proof of Theorem 6. Firstly, we show that $A: \widetilde{P} \rightarrow \widetilde{P}$ is increasing, generalized concave. For any $x \in \widetilde{P}$,

$$
\begin{aligned}
A x(t)= & -\int_{0}^{1} G(t, s) f(s, x(s)) d s-(1+t) \sum_{k=1}^{m} I_{k}\left(x\left(t_{k}\right)\right) \\
& +\sum_{0<t_{k}<t} I_{k}\left(x\left(t_{k}\right)\right)+\sum_{0<t_{k}<t}\left(t-t_{k}\right) \bar{I}_{k}\left(x\left(t_{k}\right)\right) \\
& +(1+t) \sum_{k=1}^{m} t_{k} \bar{I}_{k}\left(x\left(t_{k}\right)\right) \\
= & -\int_{0}^{1} G(t, s) f(s, x(s)) d s+\sum_{0<t_{k}<t} I_{k}\left(x\left(t_{k}\right)\right) \\
& -(1+t)\left[\sum_{0<t_{k}<t} I_{k}\left(x\left(t_{k}\right)\right)+\sum_{t \leq t_{k}<1} I_{k}\left(x\left(t_{k}\right)\right)\right] \\
& +\sum_{0<t_{k}<t}\left(t-t_{k}\right) \bar{I}_{k}\left(x\left(t_{k}\right)\right)+(1+t) \sum_{k=1}^{m} t_{k} \bar{I}_{k}\left(x\left(t_{k}\right)\right) \\
= & -\int_{0}^{1} G(t, s) f(s, x(s)) d s \\
& -\left[t \sum_{0<t_{k}<t} I_{k}\left(x\left(t_{k}\right)\right)+(1+t) \sum_{t \leq t_{k}<1} I_{k}\left(x\left(t_{k}\right)\right)\right] \\
& +\sum_{0<t_{k}<t}\left(t-t_{k}\right) \bar{I}_{k}\left(x\left(t_{k}\right)\right)+(1+t) \sum_{k=1}^{m} t_{k} \bar{I}_{k}\left(x\left(t_{k}\right)\right) .
\end{aligned}
$$

From $\left(H_{1}\right)$ and $\left(H_{2}\right)$, we know that $f(t, x(s)) \leq 0, I_{k}\left(x\left(t_{k}\right)\right) \leq$ 0 , and $\bar{I}_{k}\left(x\left(t_{k}\right)\right) \geq 0$. So we have $A x(t) \geq 0$ for $x \in \widetilde{P}$. By Lemma $4, A: \widetilde{P} \rightarrow \widetilde{P}$. It follows from $\left(H_{1}\right)$ and $\left(H_{2}\right)$ that $A$ : $\widetilde{P} \rightarrow \widetilde{P}$ is increasing. Now we prove that $A: \widetilde{P} \rightarrow \widetilde{P}$ is generalized concave. Set $\alpha(t)=\min \left\{\alpha_{1}(t), \alpha_{2}(t), \alpha_{3}(t)\right\}, t \in(0,1)$.
Then $\alpha(t) \in(t, 1)$. For any $x \in \widetilde{P}$ and $\lambda \in(0,1)$, from $\left(H_{3}\right)$ we have

$$
\begin{aligned}
& A(\lambda x)(t)=-\int_{0}^{1} G(t, s) f(s, \lambda x(s)) d s \\
& -\left[t \sum_{0<t_{k}<t} I_{k}\left(\lambda x\left(t_{k}\right)\right)+(1+t) \sum_{t \leq t_{k}<1} I_{k}\left(\lambda x\left(t_{k}\right)\right)\right] \\
& +\sum_{0<t_{k}<t}\left(t-t_{k}\right) \bar{I}_{k}\left(\lambda x\left(t_{k}\right)\right) \\
& +(1+t) \sum_{k=1}^{m} t_{k} \bar{I}_{k}\left(\lambda x\left(t_{k}\right)\right) \\
& \geq \alpha_{1}(\lambda)\left[-\int_{0}^{1} G(t, s) f(s, x(s)) d s\right]+\alpha_{2}(\lambda) \\
& \times\left[-t \sum_{0<t_{k}<t} I_{k}\left(x\left(t_{k}\right)\right)-(1+t) \sum_{t \leq t_{k}<1} I_{k}\left(x\left(t_{k}\right)\right)\right] \\
& +\alpha_{3}(\lambda)\left[\sum_{0<t_{k}<t}\left(t-t_{k}\right) \bar{I}_{k}\left(x\left(t_{k}\right)\right)\right. \\
& \left.+(1+t) \sum_{k=1}^{m} t_{k} \bar{I}_{k}\left(x\left(t_{k}\right)\right)\right] \\
& \geq \alpha(\lambda)\left\{-\int_{0}^{1} G(t, s) f(s, x(s)) d s\right. \\
& -\left[t \sum_{0<t_{k}<t} I_{k}\left(x\left(t_{k}\right)\right)\right. \\
& \left.+(1+t) \sum_{t \leq t_{k}<1} I_{k}\left(x\left(t_{k}\right)\right)\right] \\
& +\sum_{0<t_{k}<t}\left(t-t_{k}\right) \bar{I}_{k}\left(x\left(t_{k}\right)\right) \\
& \left.+(1+t) \sum_{k=1}^{m} t_{k} \bar{I}_{k}\left(x\left(t_{k}\right)\right)\right\} \\
& =\alpha(\lambda) A x(t) .
\end{aligned}
$$

That is, $A(\lambda x) \geq \alpha(\lambda) A x, x \in \widetilde{P}, \lambda \in(0,1)$.

Secondly, we prove that $A h \in \widetilde{P}_{h}$. Set

$$
r_{1}=\min _{t \in[0,1]}\left[-f\left(t, \frac{1}{2}\right)\right], \quad r_{2}=\max _{t \in[0,1]}\left[-f\left(t, \frac{3}{2}\right)\right] .
$$

Then from $\left(H_{1}\right)$, we have $r_{2} \geq r_{1}>0$. Further, from $\left(H_{1}\right),\left(H_{2}\right)$, and $\left(H_{4}\right)$,

$$
\begin{aligned}
A h(t)= & -\int_{0}^{1} G(t, s) f(s, h(s)) d s-(1+t) \sum_{k=1}^{m} I_{k}\left(h\left(t_{k}\right)\right) \\
& +\sum_{0<t_{k}<t} I_{k}\left(h\left(t_{k}\right)\right)+\sum_{0<t_{k}<t}\left(t-t_{k}\right) \bar{I}_{k}\left(h\left(t_{k}\right)\right) \\
& +(1+t) \sum_{k=1}^{m} t_{k} \bar{I}_{k}\left(h\left(t_{k}\right)\right)
\end{aligned}
$$




$$
\begin{aligned}
\geq & -\int_{0}^{1} G(t, s) f\left(s, \frac{1}{2}\right) d s \\
\geq & r_{1} \int_{0}^{1} G(t, s) d s=r_{1} h(t), \\
A h(t)= & -\int_{0}^{1} G(t, s) f(s, h(s)) d s-(1+t) \sum_{k=1}^{m} I_{k}\left(h\left(t_{k}\right)\right) \\
& +\sum_{0<t_{k}<t} I_{k}\left(h\left(t_{k}\right)\right)+\sum_{0<t_{k}<t}\left(t-t_{k}\right) \bar{I}_{k}\left(h\left(t_{k}\right)\right) \\
& +(1+t) \sum_{k=1}^{m} t_{k} \bar{I}_{k}\left(h\left(t_{k}\right)\right) \\
\leq & -\int_{0}^{1} G(t, s) f\left(s, \frac{3}{2}\right) d s-(1+t) \sum_{k=1}^{m} I_{k}\left(h\left(t_{k}\right)\right) \\
& +\sum_{k=1}^{m}\left(1-t_{k}\right) \bar{I}_{k}\left(h\left(t_{k}\right)\right)+2 \sum_{k=1}^{m} t_{k} \bar{I}_{k}\left(h\left(t_{k}\right)\right) \\
\leq & r_{2} h(t)+2\left(-\sum_{k=1}^{m} I_{k}\left(\frac{3}{2}\right)\right)+\sum_{k=1}^{m}\left(1+t_{k}\right) \bar{I}_{k}\left(\frac{3}{2}\right) \\
= & r_{2} h(t)+\sum_{k=1}^{m}\left[-2 I_{k}\left(\frac{3}{2}\right)+\left(1+t_{k}\right) \bar{I}_{k}\left(\frac{3}{2}\right)\right] \\
\leq & r_{2} h(t)+2 \sum_{k=1}^{m}\left[-2 I_{k}\left(\frac{3}{2}\right)+\left(1+t_{k}\right) \bar{I}_{k}\left(\frac{3}{2}\right)\right] \cdot h(t) \\
= & \left(r_{2}+2 \sum_{k=1}^{m}\left[-2 I_{k}\left(\frac{3}{2}\right)+\left(1+t_{k}\right) \bar{I}_{k}\left(\frac{3}{2}\right)\right]\right) h(t) .
\end{aligned}
$$

Hence,

$$
r_{1} h \leq A h \leq\left(r_{2}+2 \sum_{k=1}^{m}\left[-2 I_{k}\left(\frac{3}{2}\right)+\left(1+t_{k}\right) \bar{I}_{k}\left(\frac{3}{2}\right)\right]\right) h .
$$

That is, $A h \in \widetilde{P}_{h}$. Finally, an application of Theorem 1 implies that (i) there are $u_{0}, v_{0} \in \widetilde{P}_{h}$ such that $u_{0} \leq A u_{0}, A v_{0} \leq v_{0}$, (ii) operator equation $x=A x$ has a unique solution in $\widetilde{P}_{h}$. That is,

$$
\begin{aligned}
u_{0}(t) \leq & -\int_{0}^{1} G(t, s) f\left(s, u_{0}(s)\right) d s-(1+t) \sum_{k=1}^{m} I_{k}\left(u_{0}\left(t_{k}\right)\right) \\
& +\sum_{0<t_{k}<t} I_{k}\left(u_{0}\left(t_{k}\right)\right)+\sum_{0<t_{k}<t}\left(t-t_{k}\right) \bar{I}_{k}\left(u_{0}\left(t_{k}\right)\right) \\
& +(1+t) \sum_{k=1}^{m} t_{k} \bar{I}_{k}\left(u_{0}\left(t_{k}\right)\right), \quad t \in J, \\
v_{0}(t) \geq & -\int_{0}^{1} G(t, s) f\left(s, v_{0}(s)\right) d s \\
& -(1+t) \sum_{k=1}^{m} I_{k}\left(v_{0}\left(t_{k}\right)\right)+\sum_{0<t_{k}<t} I_{k}\left(v_{0}\left(t_{k}\right)\right)
\end{aligned}
$$

$$
\begin{aligned}
& +\sum_{0<t_{k}<t}\left(t-t_{k}\right) \bar{I}_{k}\left(v_{0}\left(t_{k}\right)\right) \\
& +(1+t) \sum_{k=1}^{m} t_{k} \bar{I}_{k}\left(v_{0}\left(t_{k}\right)\right), \quad t \in J,
\end{aligned}
$$

and the problem (1) has a unique positive solution $x^{*}$ in $\widetilde{P}_{h}$. Moreover, from Lemmas 4 and 5 we know that $x^{*} \in$ $\mathrm{PC}^{1}[J, \mathbf{R}]$. Evidently, $x^{*}$ is a positive solution of the problem (1).

Theorem 8. Assume that $\left(H_{1}\right)^{\prime}-\left(H_{4}\right)^{\prime}$ hold. Then

(i) there exist $u_{0}, v_{0} \in \widetilde{P}_{h}$ such that

$$
\begin{aligned}
u_{0}(t) \leq & -\int_{0}^{1} G(t, s) f\left(s, \bar{u}_{0}(s)\right) d s-(1+t) \sum_{k=1}^{m} I_{k}\left(\bar{u}_{0}\left(t_{k}\right)\right) \\
& +\sum_{0<t_{k}<t} I_{k}\left(\bar{u}_{0}\left(t_{k}\right)\right)+\sum_{0<t_{k}<t}\left(t-t_{k}\right) \bar{I}_{k}\left(\bar{u}_{0}\left(t_{k}\right)\right) \\
& +(1+t) \sum_{k=1}^{m} t_{k} \bar{I}_{k}\left(\bar{u}_{0}\left(t_{k}\right)\right), \quad t \in J, \\
v_{0}(t) \geq & -\int_{0}^{1} G(t, s) f\left(s, \bar{v}_{0}(s)\right) d s-(1+t) \sum_{k=1}^{m} I_{k}\left(\bar{v}_{0}\left(t_{k}\right)\right) \\
& +\sum_{0<t_{k}<t} I_{k}\left(\bar{v}_{0}\left(t_{k}\right)\right)+\sum_{0<t_{k}<t}\left(t-t_{k}\right) \bar{I}_{k}\left(\bar{v}_{0}\left(t_{k}\right)\right) \\
& +(1+t) \sum_{k=1}^{m} t_{k} \bar{I}_{k}\left(\bar{v}_{0}\left(t_{k}\right)\right), \quad t \in J,
\end{aligned}
$$

where

$$
\begin{aligned}
\bar{u}_{0}(t)= & -\int_{0}^{1} G(t, s) f\left(s, u_{0}(s)\right) d s-(1+t) \sum_{k=1}^{m} I_{k}\left(u_{0}\left(t_{k}\right)\right) \\
& +\sum_{0<t_{k}<t} I_{k}\left(u_{0}\left(t_{k}\right)\right)+\sum_{0<t_{k}<t}\left(t-t_{k}\right) \bar{I}_{k}\left(u_{0}\left(t_{k}\right)\right) \\
& +(1+t) \sum_{k=1}^{m} t_{k} \bar{I}_{k}\left(u_{0}\left(t_{k}\right)\right), \quad t \in J, \\
\bar{v}_{0}(t)= & -\int_{0}^{1} G(t, s) f\left(s, v_{0}(s)\right) d s-(1+t) \sum_{k=1}^{m} I_{k}\left(v_{0}\left(t_{k}\right)\right) \\
& +\sum_{0<t_{k}<t} I_{k}\left(v_{0}\left(t_{k}\right)\right)+\sum_{0<t_{k}<t}\left(t-t_{k}\right) \bar{I}_{k}\left(v_{0}\left(t_{k}\right)\right) \\
& +(1+t) \sum_{k=1}^{m} t_{k} \bar{I}_{k}\left(v_{0}\left(t_{k}\right)\right), \quad t \in J,
\end{aligned}
$$


(ii) the nonlinear impulsive problem (1) has a unique positive solution $x^{*}$ in $\widetilde{P}_{h} \cap P C^{1}[J, \mathbf{R}]$, where $h(t)=$ $(1 / 2)\left(t^{2}+t+1\right), t \in[0,1]$.

Proof. From the proof of Theorem 6, for any $x \in \widetilde{P}$,

$$
\begin{aligned}
A x(t)= & -\int_{0}^{1} G(t, s) f(s, x(s)) d s \\
& -\left[t \sum_{0<t_{k}<t} I_{k}\left(x\left(t_{k}\right)\right)+(1+t) \sum_{t \leq t_{k}<1} I_{k}\left(x\left(t_{k}\right)\right)\right] \\
& +\sum_{0<t_{k}<t}\left(t-t_{k}\right) \bar{I}_{k}\left(x\left(t_{k}\right)\right)+(1+t) \sum_{k=1}^{m} t_{k} \bar{I}_{k}\left(x\left(t_{k}\right)\right) .
\end{aligned}
$$

From $\left(H_{1}\right)^{\prime}$ and $\left(H_{2}\right)^{\prime}$, we know that $A x(t) \geq 0, t \in[0,1]$. By Lemma $4, A: \widetilde{P} \rightarrow \widetilde{P}$. It follows from $\left(H_{1}\right)^{\prime}$ and $\left(H_{2}\right)^{\prime}$ that $A: \widetilde{P} \rightarrow \widetilde{P}$ is decreasing. Set $\beta(t)=\max \left\{\beta_{1}(t), \beta_{2}(t), \beta_{3}(t)\right\}$, $t \in(0,1)$. Then $\beta(t) \in(0,1)$. For any $x \in \widetilde{P}$ and $\lambda \in(0,1)$, from $\left(H_{3}\right)^{\prime}$ we have

$$
\begin{aligned}
& A(\lambda x)(t)=-\int_{0}^{1} G(t, s) f(s, \lambda x(s)) d s \\
& -\left[t \sum_{0<t_{k}<t} I_{k}\left(\lambda x\left(t_{k}\right)\right)+(1+t) \sum_{t \leq t_{k}<1} I_{k}\left(\lambda x\left(t_{k}\right)\right)\right] \\
& +\sum_{0<t_{k}<t}\left(t-t_{k}\right) \bar{I}_{k}\left(\lambda x\left(t_{k}\right)\right) \\
& +(1+t) \sum_{k=1}^{m} t_{k} \bar{I}_{k}\left(\lambda x\left(t_{k}\right)\right) \\
& \leq \lambda^{-\beta_{1}(\lambda)}\left[-\int_{0}^{1} G(t, s) f(s, x(s)) d s\right]+\lambda^{-\beta_{2}(\lambda)} \\
& \times\left[-t \sum_{0<t_{k}<t} I_{k}\left(x\left(t_{k}\right)\right)-(1+t) \sum_{t \leq t_{k}<1} I_{k}\left(x\left(t_{k}\right)\right)\right] \\
& +\lambda^{-\beta_{3}(\lambda)}\left[\sum_{0<t_{k}<t}\left(t-t_{k}\right) \bar{I}_{k}\left(x\left(t_{k}\right)\right)\right. \\
& \left.+(1+t) \sum_{k=1}^{m} t_{k} \bar{I}_{k}\left(x\left(t_{k}\right)\right)\right] \\
& \leq \lambda^{-\beta(\lambda)}\left\{-\int_{0}^{1} G(t, s) f(s, x(s)) d s\right. \\
& -\left[t \sum_{0<t_{k}<t} I_{k}\left(x\left(t_{k}\right)\right)\right. \\
& \left.+(1+t) \sum_{t \leq t_{k}<1} I_{k}\left(x\left(t_{k}\right)\right)\right] \\
& +\sum_{0<t_{k}<t}\left(t-t_{k}\right) \bar{I}_{k}\left(x\left(t_{k}\right)\right)
\end{aligned}
$$

$$
\begin{aligned}
& \left.+(1+t) \sum_{k=1}^{m} t_{k} \bar{I}_{k}\left(x\left(t_{k}\right)\right)\right\} \\
& =\lambda^{-\beta(\lambda)} A x(t) .
\end{aligned}
$$

That is, $A(\lambda x) \leq \lambda^{-\beta(\lambda)} A x, x \in \widetilde{P}, \lambda \in(0,1)$. Further, for $\lambda \in(0,1)$ and $x \in \widetilde{P}$,

$$
A x=A\left(\lambda \cdot \frac{1}{\lambda} x\right) \leq \lambda^{-\beta(\lambda)} A\left(\frac{1}{\lambda} x\right) .
$$

So we obtain $A((1 / \lambda) x) \geq \lambda^{\beta(\lambda)} A x, x \in \widetilde{P}, \lambda \in(0,1)$. Consequently, $A^{2}: \widetilde{P} \rightarrow \widetilde{P}$ is increasing, and, for $x \in \widetilde{P}, \lambda \in$ $(0,1)$,

$$
\begin{aligned}
A^{2}(\lambda x) & =A(A(\lambda x)) \geq A\left(\lambda^{-\beta(\lambda)} A x\right)=A\left(\frac{1}{\lambda^{\beta(\lambda)}} A x\right) \\
& \geq\left(\lambda^{\beta(\lambda)}\right)^{\beta\left(\lambda^{\beta(\lambda)}\right)} A^{2} x \geq \lambda^{\beta(\lambda)} A^{2} x .
\end{aligned}
$$

Let $\alpha(t)=t^{\beta(t)}, t \in(0,1)$. Then $\alpha(t) \in(t, 1)$ and $A^{2}(\lambda x) \geq \alpha(\lambda) A^{2} x, x \in \widetilde{P}, \lambda \in(0,1)$. So the operator $A^{2}: \widetilde{P} \rightarrow \widetilde{P}$ is generalized concave. Next we prove that $A^{2} h \in \widetilde{P}_{h}$. Set

$$
r_{1}=\min _{t \in[0,1]}\left[-f\left(t, \frac{3}{2}\right)\right], \quad r_{2}=\max _{t \in[0,1]}\left[-f\left(t, \frac{1}{2}\right)\right] .
$$

Then from $\left(H_{1}\right)^{\prime}$, we have $r_{2} \geq r_{1}>0$. Further, from $\left(H_{1}\right)^{\prime},\left(H_{2}\right)^{\prime}$, and $\left(H_{4}\right)^{\prime}$,

$$
\begin{aligned}
A h(t)=- & \int_{0}^{1} G(t, s) f(s, h(s)) d s \\
& -(1+t) \sum_{k=1}^{m} I_{k}\left(h\left(t_{k}\right)\right)+\sum_{0<t_{k}<t} I_{k}\left(h\left(t_{k}\right)\right) \\
& +\sum_{0<t_{k}<t}\left(t-t_{k}\right) \bar{I}_{k}\left(h\left(t_{k}\right)\right)+(1+t) \sum_{k=1}^{m} t_{k} \bar{I}_{k}\left(h\left(t_{k}\right)\right) \\
\geq- & \int_{0}^{1} G(t, s) f\left(s, \frac{3}{2}\right) d s \geq r_{1} \int_{0}^{1} G(t, s) d s=r_{1} h(t), \\
A h(t)=- & \int_{0}^{1} G(t, s) f(s, h(s)) d s \\
& -(1+t) \sum_{k=1}^{m} I_{k}\left(h\left(t_{k}\right)\right)+\sum_{0<t_{k}<t} I_{k}\left(h\left(t_{k}\right)\right) \\
& +\sum_{0<t_{k}<t}\left(t-t_{k}\right) \bar{I}_{k}\left(h\left(t_{k}\right)\right) \\
& +(1+t) \sum_{k=1}^{m} t_{k} \bar{I}_{k}\left(h\left(t_{k}\right)\right) \\
\leq- & \int_{0}^{1} G(t, s) f\left(s, \frac{1}{2}\right) d s-(1+t) \sum_{k=1}^{m} I_{k}\left(h\left(t_{k}\right)\right) \\
+ & \sum_{k=1}^{m}\left(1-t_{k}\right) \bar{I}_{k}\left(h\left(t_{k}\right)\right)+2 \sum_{k=1}^{m} t_{k} \bar{I}_{k}\left(h\left(t_{k}\right)\right)
\end{aligned}
$$




$$
\begin{aligned}
& \leq r_{2} h(t)+2\left(-\sum_{k=1}^{m} I_{k}\left(\frac{1}{2}\right)\right)+\sum_{k=1}^{m}\left(1+t_{k}\right) \bar{I}_{k}\left(\frac{1}{2}\right) \\
& =r_{2} h(t)+\sum_{k=1}^{m}\left[-2 I_{k}\left(\frac{1}{2}\right)+\left(1+t_{k}\right) \bar{I}_{k}\left(\frac{1}{2}\right)\right] \\
& \leq r_{2} h(t)+2 \sum_{k=1}^{m}\left[-2 I_{k}\left(\frac{1}{2}\right)+\left(1+t_{k}\right) \bar{I}_{k}\left(\frac{1}{2}\right)\right] \cdot h(t) \\
& =\left(r_{2}+2 \sum_{k=1}^{m}\left[-2 I_{k}\left(\frac{1}{2}\right)+\left(1+t_{k}\right) \bar{I}_{k}\left(\frac{1}{2}\right)\right]\right) h(t) .
\end{aligned}
$$

Hence,

$$
r_{1} h \leq A h \leq\left(r_{2}+2 \sum_{k=1}^{m}\left[-2 I_{k}\left(\frac{1}{2}\right)+\left(1+t_{k}\right) \bar{I}_{\mathrm{k}}\left(\frac{1}{2}\right)\right]\right) h .
$$

We can choose a sufficiently small number $r_{0} \in(0,1)$ such that

$$
\begin{aligned}
r_{0} & \leq r_{1}<r_{2}+2 \sum_{k=1}^{m}\left[-2 I_{k}\left(\frac{1}{2}\right)+\left(1+t_{k}\right) \bar{I}_{k}\left(\frac{1}{2}\right)\right] \\
& \leq \frac{1}{r_{0}} .
\end{aligned}
$$

Then we get $r_{0} h \leq A h \leq\left(1 / r_{0}\right) h$. Further,

$$
\begin{aligned}
& A^{2} h=A(A h) \leq A\left(r_{0} h\right) \leq r_{0}{ }^{-\beta\left(r_{0}\right)} A h \leq \frac{1}{r_{0}{ }^{1+\beta\left(r_{0}\right)}} h, \\
& A^{2} h=A(A h) \geq A\left(\frac{1}{r_{0}} h\right) \geq r_{0}{ }^{\beta\left(r_{0}\right)} A h \geq r_{0}{ }^{1+\beta\left(r_{0}\right)} h .
\end{aligned}
$$

That is, $A^{2} h \in \widetilde{P}_{h}$. Finally, an application of Theorem 1 implies that (i) there are $u_{0}, v_{0} \in \widetilde{P}_{h}$ such that $u_{0} \leq A^{2} u_{0}, A^{2} v_{0} \leq v_{0}$, (ii) operator equation $x=A^{2} x$ has a unique solution in $\widetilde{P}_{h}$. Let $\bar{u}_{0}=A u_{0}, \bar{v}_{0}=A v_{0}$. Then, $u_{0} \leq A \bar{u}_{0}, A \bar{v}_{0} \leq v_{0}$. That is,

$$
\begin{aligned}
u_{0}(t) \leq & -\int_{0}^{1} G(t, s) f\left(s, \bar{u}_{0}(s)\right) d s \\
& -(1+t) \sum_{k=1}^{m} I_{k}\left(\bar{u}_{0}\left(t_{k}\right)\right)+\sum_{0<t_{k}<t} I_{k}\left(\bar{u}_{0}\left(t_{k}\right)\right) \\
& +\sum_{0<t_{k}<t}\left(t-t_{k}\right) \bar{I}_{k}\left(\bar{u}_{0}\left(t_{k}\right)\right) \\
& +(1+t) \sum_{k=1}^{m} t_{k} \bar{I}_{k}\left(\bar{u}_{0}\left(t_{k}\right)\right), \quad t \in J, \\
v_{0}(t) \geq & -\int_{0}^{1} G(t, s) f\left(s, \bar{v}_{0}(s)\right) d s \\
& -(1+t) \sum_{k=1}^{m} I_{k}\left(\bar{v}_{0}\left(t_{k}\right)\right)+\sum_{0<t_{k}<t} I_{k}\left(\bar{v}_{0}\left(t_{k}\right)\right) \\
& +\sum_{0<t_{k}<t}\left(t-t_{k}\right) \bar{I}_{k}\left(\bar{v}_{0}\left(t_{k}\right)\right) \\
& +(1+t) \sum_{k=1}^{m} t_{k} \bar{I}_{k}\left(\bar{v}_{0}\left(t_{k}\right)\right), \quad t \in J .
\end{aligned}
$$

Next we show that $x^{*}$ is the unique fixed point of $A$ in $\widetilde{P}_{h}$. In view of $A^{2}\left(A x^{*}\right)=A\left(A^{2} x^{*}\right)=A x^{*}$, and by the uniqueness of solutions for the operator equation $x=A^{2} x$, we have $A x^{*}=$ $x^{*}$. Suppose that $y^{*}$ is another fixed point of $A$ in $\widetilde{P}_{h}$. Then $A^{2} y^{*}=A\left(A\left(y^{*}\right)\right)=A y^{*}=y^{*}$. Hence, by the uniqueness of solutions for the operator equation $x=A^{2} x$, we obtain $x^{*}=y^{*}$. So the problem (1) has a unique positive solution $x^{*}$ in $\widetilde{P}_{h}$. Moreover, from Lemmas 4 and 5 we know that $x^{*} \in$ $\mathrm{PC}^{1}[J, \mathbf{R}]$. Evidently, $x^{*}$ is a positive solution of the problem (1).

Remark 9. Here, we provide an alternative approach to study the same type of problems under different conditions. Our result can guarantee the existence of a unique positive solution without supposing the existence of upper-lower solutions. The method used in this paper is relatively new to the literature and so is the existence and uniqueness result to the impulsive differential equations.

In the following we consider two special cases of the problem (1):

$$
\begin{gathered}
-x^{\prime \prime}(t)=f(t, x(t)), \quad t \neq t_{k}, \quad k=1,2, \ldots, m, \\
\left.\Delta x\right|_{t=t_{k}}=I_{k}\left(x\left(t_{k}\right)\right), \quad k=1,2, \ldots, m, \\
x(0)=x^{\prime}(0), \quad x(1)=x^{\prime}(1), \\
-x^{\prime \prime}(t)=f(t, x(t)), \quad t \neq t_{k}, \quad k=1,2, \ldots, m, \\
\left.\Delta x^{\prime}\right|_{t=t_{k}}=\bar{I}_{k}\left(x\left(t_{k}\right)\right), \quad k=1,2, \ldots, m, \\
x(0)=x^{\prime}(0), \quad x(1)=x^{\prime}(1),
\end{gathered}
$$

where $f \in C[J \times \mathbf{R}, \mathbf{R}], I_{k}, \bar{I}_{k} \in C[\mathbf{R}, \mathbf{R}], k=1,2, \ldots, m$.

From Theorems 6 and 8 , we have the following conclusions.

Corollary 10. Assume that $\left(H_{1}\right)$ holds and

$\left(G_{1}\right) I_{k}(0) \leq 0$ and $I_{k}(x)$ is decreasing in $x \in[0, \infty), k=$ $1,2, \ldots, m$ with

$$
\sum_{k=1}^{m} I_{k}\left(\frac{3}{2}\right)<0
$$

$\left(G_{2}\right)$ for any $\lambda \in(0,1), t \in[0,1]$ and $x \geq 0$, there exist $\alpha_{1}(\lambda), \alpha_{2}(\lambda) \in(\lambda, 1)$ such that

$$
\begin{aligned}
& f(t, \lambda x) \leq \alpha_{1}(\lambda) f(t, x) \\
& I_{k}(\lambda x) \leq \alpha_{2}(\lambda) I_{k}(x), \quad k=1,2, \ldots, m
\end{aligned}
$$

Then

(i) there exist $u_{0}, v_{0} \in \widetilde{P}_{h}$ such that

$$
\begin{aligned}
u_{0}(t) \leq & -\int_{0}^{1} G(t, s) f\left(s, u_{0}(s)\right) d s \\
& -(1+t) \sum_{k=1}^{m} I_{k}\left(u_{0}\left(t_{k}\right)\right)+\sum_{0<t_{k}<t} I_{k}\left(u_{0}\left(t_{k}\right)\right), \quad t \in J,
\end{aligned}
$$




$$
\begin{aligned}
v_{0}(t) \geq & -\int_{0}^{1} G(t, s) f\left(s, v_{0}(s)\right) d s \\
& -(1+t) \sum_{k=1}^{m} I_{k}\left(v_{0}\left(t_{k}\right)\right)+\sum_{0<t_{k}<t} I_{k}\left(v_{0}\left(t_{k}\right)\right), \quad t \in J,
\end{aligned}
$$

(ii) the nonlinear impulsive problem (34) has a unique positive solution $x^{*}$ in $\widetilde{P}_{h}$, where $h(t)=(1 / 2)\left(t^{2}+t+1\right)$, $t \in[0,1]$, and $G(t, s)$ is given as in Lemma 4.

Corollary 11. Assume that $\left(H_{1}\right)^{\prime}$ hold and

$\left(G_{1}\right)^{\prime} I_{k}(x) \leq 0$ for $[0, \infty)$ and $I_{k}(x)$ is increasing in $x \in$ $[0, \infty), k=1,2, \ldots, m$ with

$$
\sum_{k=1}^{m} I_{k}\left(\frac{1}{2}\right)<0
$$

$\left(G_{2}\right)^{\prime}$ for any $\lambda \in(0,1), t \in[0,1]$ and $x \geq 0$, there exist $\beta_{1}(\lambda), \beta_{2}(\lambda) \in(0,1)$ such that

$$
\begin{aligned}
& f(t, \lambda x) \geq \lambda^{-\beta_{1}(\lambda)} f(t, x), \\
& I_{k}(\lambda x) \geq \lambda^{-\beta_{2}(\lambda)} I_{k}(x), \quad k=1,2, \ldots, m .
\end{aligned}
$$

Then

(i) there exist $u_{0}, v_{0} \in \widetilde{P}_{h}$ such that

$$
\begin{aligned}
u_{0}(t) \leq & -\int_{0}^{1} G(t, s) f\left(s, \bar{u}_{0}(s)\right) d s-(1+t) \sum_{k=1}^{m} I_{k}\left(\bar{u}_{0}\left(t_{k}\right)\right) \\
& +\sum_{0<t_{k}<t} I_{k}\left(\bar{u}_{0}\left(t_{k}\right)\right), \quad t \in J, \\
v_{0}(t) \geq & -\int_{0}^{1} G(t, s) f\left(s, \bar{v}_{0}(s)\right) d s \\
& -(1+t) \sum_{k=1}^{m} I_{k}\left(\bar{v}_{0}\left(t_{k}\right)\right)+\sum_{0<t_{k}<t} I_{k}\left(\bar{v}_{0}\left(t_{k}\right)\right), \quad t \in J,
\end{aligned}
$$

where

$$
\begin{aligned}
\bar{u}_{0}(t)= & -\int_{0}^{1} G(t, s) f\left(s, u_{0}(s)\right) d s \\
& -(1+t) \sum_{k=1}^{m} I_{k}\left(u_{0}\left(t_{k}\right)\right) \\
& +\sum_{0<t_{k}<t} I_{k}\left(u_{0}\left(t_{k}\right)\right), \quad t \in J, \\
\bar{v}_{0}(t)= & -\int_{0}^{1} G(t, s) f\left(s, v_{0}(s)\right) d s \\
& -(1+t) \sum_{k=1}^{m} I_{k}\left(v_{0}\left(t_{k}\right)\right) \\
& +\sum_{0<t_{k}<t} I_{k}\left(v_{0}\left(t_{k}\right)\right), \quad t \in J,
\end{aligned}
$$

(ii) the nonlinear impulsive problem (34) has a unique positive solution $x^{*}$ in $\widetilde{P}_{h}$, where $h(t)=(1 / 2)\left(t^{2}+t+1\right)$ and $t \in[0,1]$ and $G(t, s)$ is given as in Lemma 4 .

Corollary 12. Assume that $\left(H_{1}\right)$ holds and

$\left(G_{3}\right) \bar{I}_{k}(0) \geq 0$ and $\bar{I}_{k}(x)$ is increasing in $x \in[0, \infty), k=$ $1,2, \ldots, m$ with

$$
\sum_{k=1}^{m}\left[\left(1+t_{k}\right) \bar{I}_{k}\left(\frac{3}{2}\right)\right]>0,
$$

$\left(G_{4}\right)$ for any $\lambda \in(0,1), t \in[0,1]$ and $x \geq 0$, there exist $\alpha_{1}(\lambda), \alpha_{2}(\lambda) \in(\lambda, 1)$ such that

$$
\begin{aligned}
& f(t, \lambda x) \leq \alpha_{1}(\lambda) f(t, x), \\
& \bar{I}_{k}(\lambda x) \geq \alpha_{2}(\lambda) \bar{I}_{k}(x), \quad k=1,2, \ldots, m .
\end{aligned}
$$

Then

(i) there exist $u_{0}, v_{0} \in \breve{P}_{h}$ such that

$$
\begin{aligned}
u_{0}(t) \leq & -\int_{0}^{1} G(t, s) f\left(s, u_{0}(s)\right) d s \\
& +\sum_{0<t_{k}<t}\left(t-t_{k}\right) \bar{I}_{k}\left(u_{0}\left(t_{k}\right)\right) \\
& +(1+t) \sum_{k=1}^{m} t_{k} \bar{I}_{k}\left(u_{0}\left(t_{k}\right)\right), \quad t \in J, \\
v_{0}(t) \geq & -\int_{0}^{1} G(t, s) f\left(s, v_{0}(s)\right) d s \\
& +\sum_{0<t_{k}<t}\left(t-t_{k}\right) \bar{I}_{k}\left(v_{0}\left(t_{k}\right)\right) \\
& +(1+t) \sum_{k=1}^{m} t_{k} \bar{I}_{k}\left(v_{0}\left(t_{k}\right)\right), \quad t \in J,
\end{aligned}
$$

(ii) the nonlinear impulsive problem (35) has a unique positive solution $x^{*}$ in $\breve{P}_{h} \cap P C^{1}[J, \mathbf{R}]$, where $h(t)=$ $(1 / 2)\left(t^{2}+t+1\right), t \in[0,1], \breve{P}=\{x \in C[J, \mathbf{R}] \mid$ $x(t) \geq 0, t \in J\}$, and $P C^{1}[J, \mathbf{R}]=\left\{x \in C[J, \mathbf{R}] \mid x^{\prime}(t)\right.$ is continuous at $t \neq t_{k}$ and left continuous at $t=t_{k}$, $x^{\prime}\left(t_{k}^{+}\right)$exists, $\left.k=1,2, \ldots, m\right\}$.

Corollary 13. Assume that $\left(H_{1}\right)^{\prime}$ hold and

$\left(G_{3}\right)^{\prime} \bar{I}_{k}(x) \geq 0$ for $[0, \infty)$ and $\bar{I}_{k}(x)$ is decreasing in $x \in$ $[0, \infty), k=1,2, \ldots, m$ with

$$
\sum_{k=1}^{m}\left(1+t_{k}\right) \bar{I}_{k}\left(\frac{1}{2}\right)>0,
$$

$\left(G_{4}\right)^{\prime}$ for any $\lambda \in(0,1), t \in[0,1]$, and $x \geq 0$, there exist $\beta_{1}(\lambda), \beta_{2}(\lambda) \in(0,1)$ such that

$$
\begin{aligned}
& f(t, \lambda x) \geq \lambda^{-\beta_{1}(\lambda)} f(t, x), \\
& \bar{I}_{k}(\lambda x) \leq \lambda^{-\beta_{2}(\lambda)} \bar{I}_{k}(x), \quad k=1,2, \ldots, m .
\end{aligned}
$$


Then

(i) there exist $u_{0}, v_{0} \in \breve{P}_{h}$ such that

$$
\begin{aligned}
u_{0}(t) \leq & -\int_{0}^{1} G(t, s) f\left(s, \bar{u}_{0}(s)\right) d s \\
& +\sum_{0<t_{k}<t}\left(t-t_{k}\right) \bar{I}_{k}\left(\bar{u}_{0}\left(t_{k}\right)\right) \\
& +(1+t) \sum_{k=1}^{m} t_{k} \bar{I}_{k}\left(\bar{u}_{0}\left(t_{k}\right)\right), \quad t \in J, \\
v_{0}(t) \geq & -\int_{0}^{1} G(t, s) f\left(s, \bar{v}_{0}(s)\right) d s \\
& +\sum_{0<t_{k}<t}\left(t-t_{k}\right) \bar{I}_{k}\left(\bar{v}_{0}\left(t_{k}\right)\right) \\
& +(1+t) \sum_{k=1}^{m} t_{k} \bar{I}_{k}\left(\bar{v}_{0}\left(t_{k}\right)\right), \quad t \in J,
\end{aligned}
$$

where

$$
\begin{aligned}
\bar{u}_{0}(t)= & -\int_{0}^{1} G(t, s) f\left(s, u_{0}(s)\right) d s \\
& +\sum_{0<t_{k}<t}\left(t-t_{k}\right) \bar{I}_{k}\left(u_{0}\left(t_{k}\right)\right) \\
& +(1+t) \sum_{k=1}^{m} t_{k} \bar{I}_{k}\left(u_{0}\left(t_{k}\right)\right), \quad t \in J, \\
\bar{v}_{0}(t)= & -\int_{0}^{1} G(t, s) f\left(s, v_{0}(s)\right) d s \\
& +\sum_{0<t_{k}<t}\left(t-t_{k}\right) \bar{I}_{k}\left(v_{0}\left(t_{k}\right)\right) \\
& +(1+t) \sum_{k=1}^{m} t_{k} \bar{I}_{k}\left(v_{0}\left(t_{k}\right)\right), \quad t \in J,
\end{aligned}
$$

(ii) the nonlinear impulsive problem (35) has a unique positive solution $x^{*}$ in $\breve{P}_{h} \bigcap P C^{1}[J, \mathbf{R}]$, where $h(t)=$ $(1 / 2)\left(t^{2}+t+1\right), t \in[0,1], \breve{P}=\{x \in C[J, \mathbf{R}] \mid$ $x(t) \geq 0, t \in J\}$, and $P C^{1}[J, \mathbf{R}]=\{x \in C[J, \mathbf{R}] \mid$ $x^{\prime}(t)$ is continuous at $t \neq t_{k}$ and left continuous at $t=$ $t_{k}, x^{\prime}\left(t_{k}^{+}\right)$exists, $\left.k=1,2, \ldots, m\right\}$.

\section{An Example}

To illustrate how our main results can be used in practice we present an example.
Example 1. Consider the following boundary value problem:

$$
\begin{gathered}
-x^{\prime \prime}(t)=-\sqrt{t x+4}, \quad t \in J, \quad t \neq \frac{1}{2}, \\
\left.\Delta x\right|_{t=1 / 2}=-x^{1 / 3}\left(\frac{1}{2}\right), \\
\left.\Delta x^{\prime}\right|_{t=1 / 2}=x^{1 / 4}\left(\frac{1}{2}\right), \\
x(0)=x^{\prime}(0), \quad x(1)=x^{\prime}(1) .
\end{gathered}
$$

Conclusion. BVP (50) has a unique positive solution in $\widetilde{P}_{h}$, where $h(t)=(1 / 2)\left(t^{2}+t+1\right), t \in[0,1]$.

Proof. BVP (50) can be regarded as a BVP of the form (1), where $t_{1}=(1 / 2), f(t, x)=-\sqrt{t x+4}, I_{1}(x)=-x^{1 / 3}$, and $\bar{I}_{1}(x)=x^{1 / 4}$. It is not difficult to see that the conditions $\left(H_{1}\right),\left(H_{2}\right)$, and $\left(H_{4}\right)$ hold. In addition, let $\alpha_{1}(\lambda)=\lambda^{1 / 2}$, $\alpha_{2}(\lambda)=\lambda^{1 / 3}$, and $\alpha_{3}(\lambda)=\lambda^{1 / 4}$. Then, the condition $\left(H_{3}\right)$ of Theorem 6 holds. Hence, by Theorem 6 , the conclusion follows, and the proof is complete.

Remark 14. Example 1 implies that there is a large number of functions that satisfy the conditions of Theorem 6. In addition, the conditions of Theorem 6 are also easy to check.

\section{Acknowledgments}

The research was supported by the Fund of National Natural Science of China $(61250011,11201272)$ and the Science Foundation of Shanxi Province (2012011004-4, 2010021002-1).

\section{References}

[1] A. Cabada and E. Liz, "Boundary value problems for higher order ordinary differential equations with impulses," Nonlinear Analysis: Theory, Methods and Applications, vol. 32, no. 6, pp. 775-786, 1998.

[2] A. Cabada, J. J. Nieto, D. Franco, and S. I. Trofimchuk, "A generalization of the monotone method for second order periodic boundary value problem with impulses at fixed points," Dynamics of Continuous, Discrete and Impulsive Systems, vol. 7, no. 1, pp. 145-158, 2000.

[3] L. Chen and J. Sun, "Boundary value problem of second order impulsive functional differential equations," Journal of Mathematical Analysis and Applications, vol. 323, no. 1, pp. 708720, 2006.

[4] W. Ding, M. Han, and J. Mi, "Periodic boundary value problem for the second-order impulsive functional differential equations," Computers \& Mathematics with Applications, vol. 50, no. 3-4, pp. 491-507, 2005.

[5] L. H. Erbe and X. Liu, "Existence results for boundary value problems of second order impulsive differential equations," Journal of Mathematical Analysis and Applications, vol. 149, no. 1, pp. 56-69, 1990.

[6] M. Feng and D. Xie, "Multiple positive solutions of multipoint boundary value problem for second-order impulsive differential equations," Journal of Computational and Applied Mathematics, vol. 223, no. 1, pp. 438-448, 2009. 
[7] D. J. Guo, "Existence of solutions of boundary value problems for nonlinear second order impulsive differential equations in Banach spaces," Journal of Mathematical Analysis and Applications, vol. 181, no. 2, pp. 407-421, 1994.

[8] L. Hu, L. Liu, and Y. Wu, "Positive solutions of nonlinear singular two-point boundary value problems for second-order impulsive differential equations," Applied Mathematics and Computation, vol. 196, no. 2, pp. 550-562, 2008.

[9] T. Jankowski, "Existence of solutions for second order impulsive differential equations with deviating arguments," Nonlinear Analysis: Theory, Methods and Applications, vol. 67, no. 6, pp. 1764-1774, 2007.

[10] T. Jankowski, "Positive solutions to second order four-point boundary value problems for impulsive differential equations," Applied Mathematics and Computation, vol. 202, no. 2, pp. 550561, 2008.

[11] T. Jankowski, "Existence of positive solutions to second order four-point impulsive differential problems with deviating arguments," Computers \& Mathematics with Applications, vol. 58, no. 4, pp. 805-817, 2009.

[12] E. K. Lee and Y.-H. Lee, "Multiple positive solutions of singular two point boundary value problems for second order impulsive differential equations," Applied Mathematics and Computation, vol. 158, no. 3, pp. 745-759, 2004.

[13] X. Lin and D. Jiang, "Multiple positive solutions of Dirichlet boundary value problems for second order impulsive differential equations," Journal of Mathematical Analysis and Applications, vol. 321, no. 2, pp. 501-514, 2006.

[14] L. Liu, L. Hu, and Y. Wu, "Positive solutions of two-point boundary value problems for systems of nonlinear secondorder singular and impulsive differential equations," Nonlinear Analysis: Theory, Methods and Applications, vol. 69, no. 11, pp. 3774-3789, 2008.

[15] E. Liz and J. J. Nieto, "The monotone iterative technique for periodic boundary value problems of second order impulsive differential equations," Commentationes Mathematicae Universitatis Carolinae, vol. 34, no. 3, pp. 405-411, 1993.

[16] J. J. Nieto and R. Rodríguez-López, "Periodic boundary value problem for non-Lipschitzian impulsive functional differential equations," Journal of Mathematical Analysis and Applications, vol. 318, no. 2, pp. 593-610, 2006.

[17] I. Rachůnková and M. Tvrdý, "Existence results for impulsive second-order periodic problems," Nonlinear Analysis: Theory, Methods and Applications, vol. 59, no. 1-2, pp. 133-146, 2004.

[18] I. Rachůnková and M. Tvrdý, "Non-ordered lower and upper functions in second order impulsive periodic problems," Dynamics of Continuous, Discrete \& Impulsive Systems A, vol. 12, no. 3-4, pp. 397-415, 2005.

[19] M. U. Akhmet, G. A. Bekmukhambetova, and Y. Serinagaoglu, The Dynamics of the Systemic Arterial Pressure Through Impulsive Differential Equations, Institute for Mathematics and its Applications, Middle East Technical University, Ankara, Turkey, 2005.

[20] A. d'Onofrio, "On pulse vaccination strategy in the SIR epidemic model with vertical transmission," Applied Mathematics Letters, vol. 18, no. 7, pp. 729-732, 2005.

[21] S. Gao, L. Chen, J. J. Nieto, and A. Torres, "Analysis of a delayed epidemic model with pulse vaccination and saturation incidence," Vaccine, vol. 24, no. 35-36, pp. 6037-6045, 2006.

[22] W.-T. Li and H.-F. Huo, "Global attractivity of positive periodic solutions for an impulsive delay periodic model of respiratory dynamics," Journal of Computational and Applied Mathematics, vol. 174, no. 2, pp. 227-238, 2005.

[23] S. Tang and L. Chen, "Density-dependent birth rate, birth pulses and their population dynamic consequences," Journal of Mathematical Biology, vol. 44, no. 2, pp. 185-199, 2002.

[24] W. Zhang and M. Fan, "Periodicity in a generalized ecological competition system governed by impulsive differential equations with delays," Mathematical and Computer Modelling, vol. 39, no. 4-5, pp. 479-493, 2004.

[25] X. Zhang, Z. Shuai, and K. Wang, "Optimal impulsive harvesting policy for single population," Nonlinear Analysis: Theory, Methods and Applications, vol. 4, no. 4, pp. 639-651, 2003.

[26] R. P. Agarwal and D. O'Regan, "A multiplicity result for second order impulsive differential equations via the Leggett Williams fixed point theorem," Applied Mathematics and Computation, vol. 161, no. 2, pp. 433-439, 2005.

[27] W. Wang, X. Fu, and X. Yang, "Positive solutions of periodic boundary value problems for impulsive differential equations," Computers \& Mathematics with Applications, vol. 58, no. 8, pp. 1623-1630, 2009.

[28] X. Zhang, "Existence of positive solution for second-order nonlinear impulsive singular differential equations of mixed type in Banach spaces," Nonlinear Analysis: Theory, Methods and Applications, vol. 70, no. 4, pp. 1620-1628, 2009.

[29] J. Chu, X. Lin, D. Jiang, D. O’Regan, and R. P. Agarwal, "Positive solutions for second-order superlinear repulsive singular Neumann boundary value problems," Positivity, vol. 12, no. 3, pp. 555-569, 2008.

[30] C.-B. Zhai, C. Yang, and X.-Q. Zhang, "Positive solutions for nonlinear operator equations and several classes of applications," Mathematische Zeitschrift, vol. 266, no. 1, pp. 43-63, 2010. 


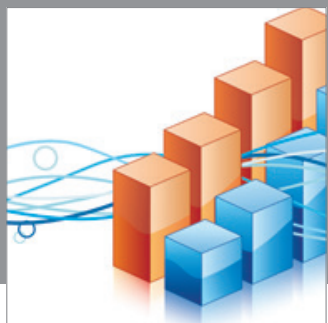

Advances in

Operations Research

mansans

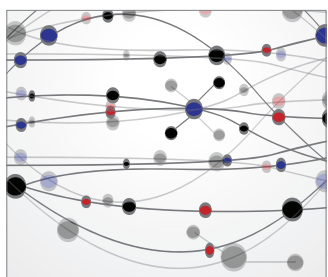

The Scientific World Journal
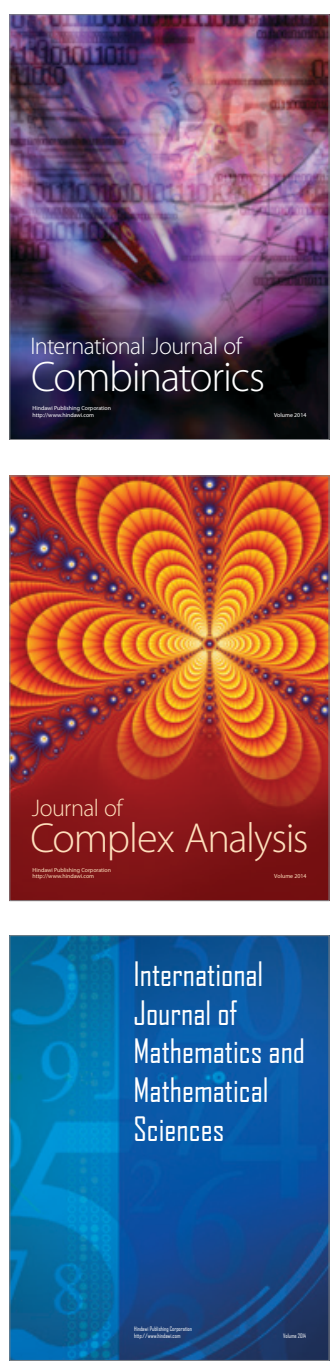
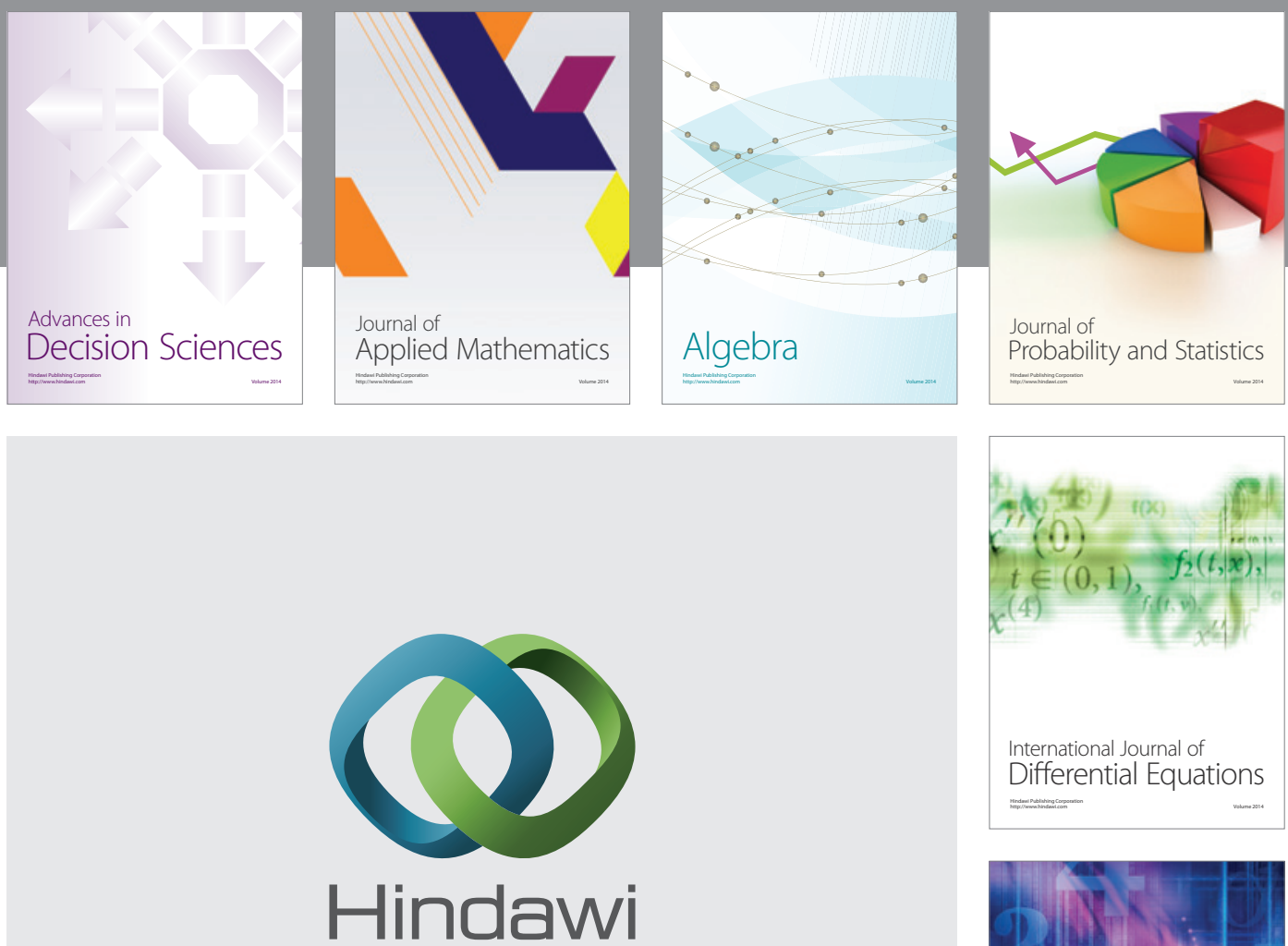

Submit your manuscripts at http://www.hindawi.com
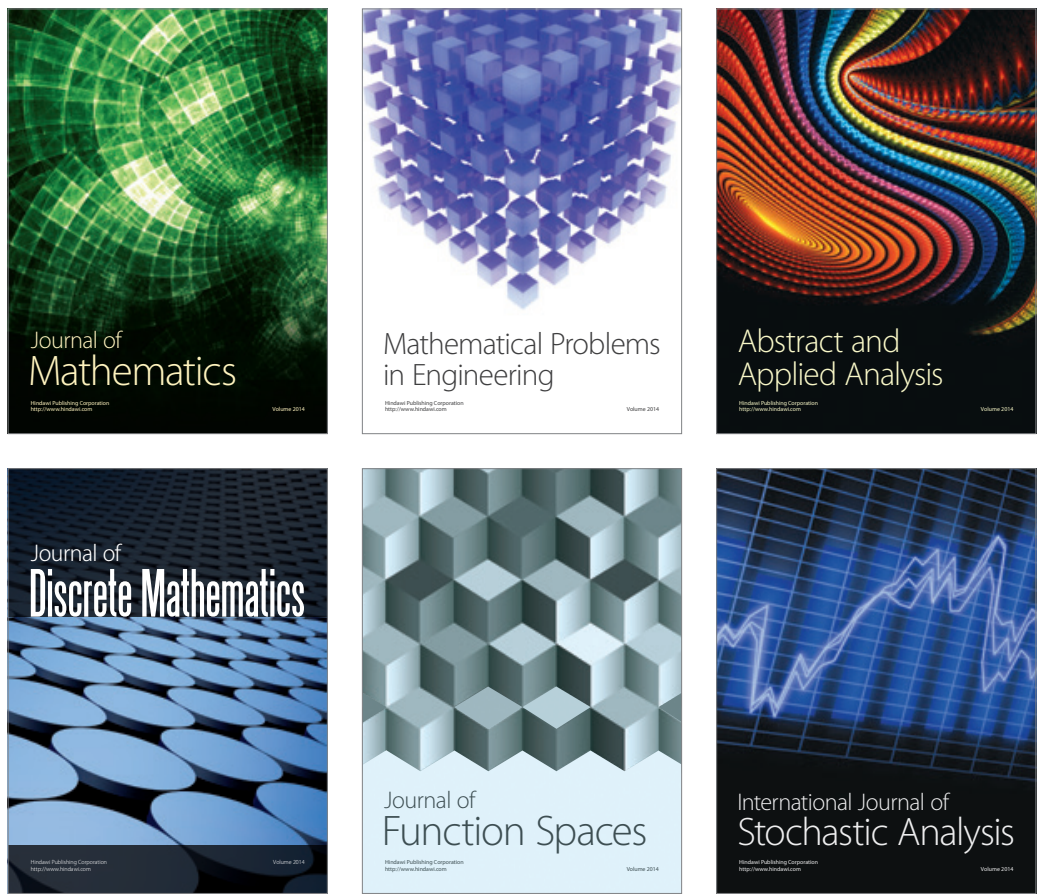

Journal of

Function Spaces

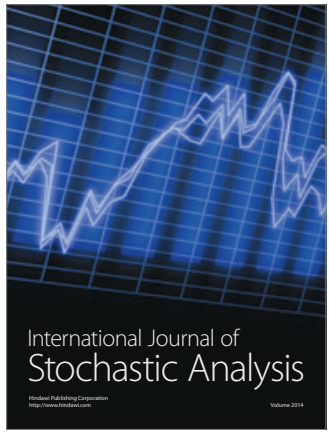

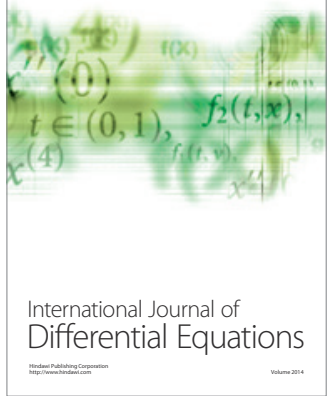
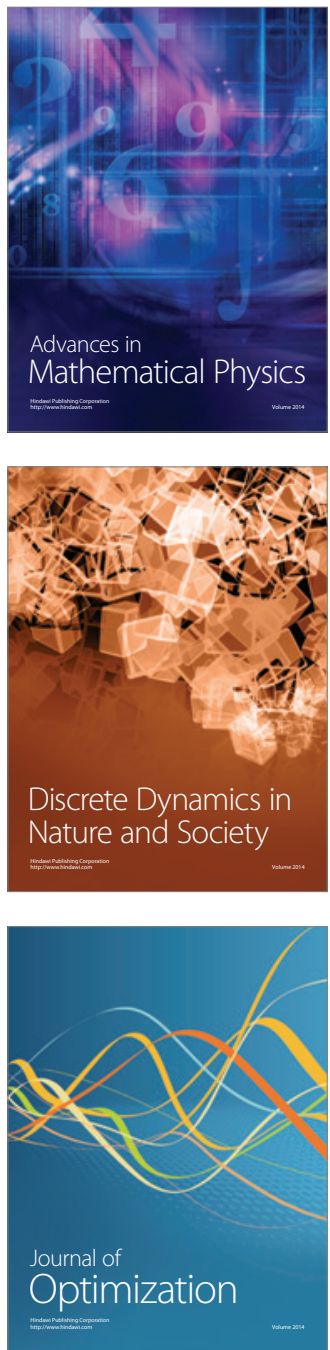